\title{
Éditorial
}

\section{Le soutien social: un processus relationnel et non une commodité}

Il y a presque vingt ans, Gerald Caplan (1974), John Cassel (1974) et Sydney Cobb (1976) ont écrit des articles traitant du soutien social qui ont largement influencé la définition du concept de soutien social, et qui ont contribué au déferlement de recherches empiriques et d'analyses conceptuelles sur le rôle des relations humaines chez les personnes dans les processus reliés au stress et à sa gestion. Chacun de ces auteurs mettait l'emphase sur un aspect particulier de la structure du soutien offert par l'entourage. Caplan a concentré ses recherches sur les fonctions de base du " réseau de soutien " à l'égard de la santé mentale, et a suggéré plusieurs moyens d'en accroître la capacité de réponse aux besoins psychosociaux des personnes, particulièrement lorsqu'elles sont aux prises avec des situations stressantes. Cassel a, pour sa part, orienté ses travaux sur l'aspect de la signification épidémiologique du soutien social en allégant que l'isolement social constituait un facteur de risque pour la santé, mais que l'intégration sociale jouait un rôle préventif. Les recherches de Cobb nous ont fourni les bases d'une définition du soutien social qui reconnaissait l'importance, du point de vue psychologique, d'être aimé et soigné, et de faire partie d'un réseau de personnes sur lesquelles on pouvait se fier. De plus, il a placé cette définition de soutien social dans un cadre de recherche conceptuelle qui est maintenant mieux connue sous le terme de modèle d'absorption du stress (stress buffering) du soutien social. Ce modèle est fondé sur l'hypothèse que, placées dans une situation de stress, les personnes qui se sentent appuyées seront mieux protégées contre les effets négatifs du stress.

Dans le cadre du présent numéro de La Revue canadienne du vieillissement, Philippe Landreville et Philippe Cappeliez examinent en détail toute la documentation relative à la relation entre le soutien social et les symptômes dépressifs chez les aînés. Ils soupèsent les données par rapport à deux versions différentes du modèle d'absorption du stress. La première démontre que le soutien du milieu contribue à diminuer la dépression grâce à son interaction avec les facteurs de stress (le modèle d'absorption), et la deuxième établit que les facteurs de stress stimulent le soutien, qui à son tour, contribue à réduire le stress (la mobilisation ou l'effet direct). Les auteurs ont comparé le modèle d'absorption avec le modèle d'effet direct qui suggère que le soutien social contribue au mieux-être en général, indépendamment du stress. Dans le contexte de la dépression, une corrélation négative demeure entre le soutien et la symptomatologie, ce qui contribue à favoriser ce dernier modèle.

Les recherches effectuées par Landreville et Cappeliez les ont amené à la conclusion que la somme des évidences recueillies favorise autant le modèle 
d'absorption du stress que le modèle d'effet direct relativement aux effets du soutien social sur la dépression. Cependant, cette conclusion est formulée avec plusieurs réserves, étant donné que très peu d'études ont jusqu'ici porté sur la vérification de ces deux modèles basés sur le même échantillonnage, que les études diffèrent largement dans l'échantillonnage des personnes âgées et des mesures de soutien, et que la majorité des études sont transversales plutôt que longitudinales. Les auteurs ont également soulevé le fait qu'il existe une confusion entre les types de mesures qui déterminent le soutien du point de vue de sa structure et ceux qui spécifient ses fonctions. Par exemple, ils ont observé que la présence d'un confident a été interprétée par certains chercheurs comme un indice de la structure du soutien, et par d'autres, comme un indice à l'effet que le répondant a accès à une certaine forme de soutien.

Landreville et Cappeliez maintiennent qu'une des raisons pour lesquelles les preuves des analyses empiriques ne favorisent pas nécessairement et pas toujours un modèle de soutien par rapport à un autre est que les analyses ne découpent pas les facteurs de stress des répondants en catégories particulières de demandes stressantes pour les répondants, et qu'elles n'examinent pas de quelle manière chaque type de soutien, provenant de différentes personnes, exerce une influence sur la gestion du stress et la santé mentale. Par conséquent, les auteurs exigent plus de précision dans l'identification des types de soutien face aux différents facteurs de stress, et des sources de soutien qui peuvent répondre à ces besoins. Ils croient que des analyses plus poussées auraient le mérite de révéler les conditions exactes sous lequelles le soutien social a un effet de temporisation en comparaison avec l'effet direct sur la santé mentale.

Permettez-moi de ne pas être de cet avis. Voici les raisons pour lesquelles je ne partage pas l'opinion de ceux qui maintiennent que les questions restées sans réponse entourant le rôle du soutien social dans le processus de stress le seront vraisemblablement dans les études qui discerneront comment les types et les sources de soutien correspondent aux demandes engendrées par différents facteurs de stress. En tout premier lieu, nous devons reconsidérer notre manière de concevoir et d'évaluer le soutien social. $\grave{A}$ l'origine, lorsque Caplan, Cassel et Cobb ont présenté leurs idées sur le concept, l'objet de leur recherche était les liens sociaux entre les personnes et les fonctions protectrices sur la santé. Ils ont avancé l'hypothèse que les membres du réseau social se fournissent mutuellement des impressions visà-vis leurs attitudes et leur estime de soi, qu'ils partagent les soucis de chacun, qu'ils s'offrent de la camaraderie, des conseils, du soutien moral et de l'aide au besoin. Mais l'accent était toujours mis sur la capacité d'un groupe social particulier à communiquer cette information et ce type de soutien. La particularité du groupe dépendait de l'importance qu'attachait la personne à l'étude aux membres du réseau. En fait, l'influence du soutien du réseau découlait de l'importance qu'attachait cette personne à sa relation avec chaque membre du réseau.

Lorsque le soutien social est devenu le sujet de nombreuses études empi- 
riques, des méthodes ont été élaborées qui ont eu pour effet de déplacer les ressources de leur propre contexte de relation. Les chercheurs ont concentré leurs travaux exclusivement sur les bénéfices découlant du réseau, en faisant une différence entre le soutien émotif, concret et de haute estime. Ils ont oublié le fait que seule l'existence d'une relation préalablement établie pouvait amener à des interactions de soutien entre les personnes. De plus, en détachant le soutien du contexte d'une relation, on en est venu à le percevoir comme une commodité d'échange et non comme la manifestation d'un des nombreux processus de l'entretien de relations humaines. Il faut également souligner que le soutien est devenu une variable pouvant être mesurée pour connaître une quantité certaine et une qualité relative, à la place d'être perçu comme un processus dynamique obéissant à un ensemble complexe de contingences qui exercent une influence sur les interactions sociales.

En demandant aux répondants d'évaluer les ressources dont ils profitent ou celles dont ils croient qu'ils pourraient bénéficier à l'intérieur du réseau, les chercheurs n'ont pas augmenté leur connaissance sur la manière dont l'interaction sociale influence la santé et le bien-être d'une personne. C'est pourquoi les investigations devraient être centrées sur les échanges mêmes liés au stress ayant lieu entre certains membres du réseau, et non sur des évaluations générales des répondants sur la quantité, la qualité ou l'efficacité du soutien. De plus, en se concentrant exclusivement sur l'aspect de soutien des échanges, les chercheurs ont raté l'occasion d'identifier d'autres processus interpersonnels qui ont de fortes chances d'influencer les comportements, les connaissances et les émotions. Par exemple, il a été prouvé que les manifestations de conflit, d'hostilité, de critique ou de surprotection peuvent neutraliser les effets protecteurs des manifestations de soutien. K.S. Rook (1984) a découvert que les conflits dans les relations personnelles avaient une plus forte incidence sur le bien-être des veuves plus âgées que le soutien. Dans le cadre de la dépression, les recherches publiées par I.H. Gotlib et J.M. Hooley (1988) révèlent de quelle manière l'évaluation des processus interactionnels multiples, non seulement le soutien, a contribué à élargir notre connaissance des liens causals entre la dépression et les difficultés conjugales.

Lorsque l'évaluation débute sur l'étude exclusive des types de ressources que les gens obtiennent des autres pour s'élargir aux processus des interactions entre les personnes dans les relations humaines plus intimes, l'étude du soutien social devient plus complexe et plus fidèle à la réalité. Elle devient plus difficile parce que les gens ont des attentes bien différentes, compte tenu du type de relation humaine, et ont des intérêts bien différents ce qui influence largement ce qu'ils retirent de leurs interactions avec chaque personne. Permettez-moi d'illustrer ceci d'un exemple. Dans une étude récente et effectuée avec M. Steinberg (Steinberg et Gottlieb, à venir), nous avons interrogé des femmes provenant de ménage à deux revenus sur le soutien qu'elles obtenaient de leur mari lorsqu'elles étaient placées dans une situation conflictuelle entre le travail et les exigences familiales. Après avoir décrit de quelle manière le mari avait collaboré en vue de régler ce conflit, 
les femmes évaluaient le niveau de soutien apporté par le conjoint en donnant une note de un à sept. Cependant, lorsque nous leur avons demandé la raison de chaque note attribuée, nous avons appris que chacune des évaluations reflétaient une moyenne fondée sur plusieurs critères. Par exemple, un mari qui s'absente de son travail pour aller chercher un enfant malade à l'école et le conduire à la maison reçoit une note très haute pour sa contribution à la résolution d'une situation d'urgence, mais reçoit un note très basse lorsque sa conjointe doit le convaincre avant qu'il décide de poser un geste et qu'elle doit payer en retour pour l'aide lorsqu'elle revient à la maison. En fait, une incidence positive sur le règlement d'une situation difficile a été contrecarrée par l'aspect désagréable d'avoir à plaider l'assistance du conjoint et d'avoir une dette envers ce dernier.

Cet exemple démontre que les interactions ne peuvent pas être divisées exclusivement en une catégorie de soutien et de non soutien, mais qu'elles peuvent contenir des éléments provenant des deux. Il révèle également que les relations humaines faussent la signification première du soutien dans les interactions, parce que les attentes dans une relation varient d'une personne à l'autre. Il faut également prendre en considération que les différents processus qui interviennent dans les différentes relations ont non seulement un impact sur l'aboutissement en modifiant la trajectoire de la gestion, mais influencent aussi la qualité et le déroulement de la relation même. Lorsqu'une femme sollicite l'aide de son époux lors d'une situation urgente qui concerne leur enfant, et lorsqu'elle doit en retour faire quelque chose pour lui par la suite, elle éprouvera vraisemblablement du ressentiment envers ce dernier et remettre en question les notions de partage et d'engagement dans le marriage. Malgré le fait que la situation difficile a été réglée, le processus de résolution et ses effets psychologiques ont peut-être engendré des tensions supplémentaires dans la relation.

Quelles sont les implications de cette persepctive envers l'étude de la relation entre le soutien social et la dépression chez les personnes âgées? $\mathrm{Si}$ les études empiriques mettaient l'accent sur les interactions qui surviennent dans les relations intimes, reconnaissant ainsi totalement le caractère social des liens humains, il n'y aurait donc plus lieu de conclure en dénonçant le manque de recherche sur le processus ou les mécanismes sousjacents des effets du soutien social sur la dépression ou sur toutes les manifestations de la santé mentale. Nous serions obligés d'effectuer des évaluations laborieuses des interactions qui ont lieu dans la conduite des relations humaines à l'aide d'entrevues plus approfondies, des relevés journaliers, des enregistrements des interactions et des procédés de codage des observations. Ces méthodes nous permettraient de percevoir le soutien social comme un processus dynamique qui fait l'objet de négociations et qui est influencé par une foule de facteurs personnels, situationnels et relationnels. Dans le cadre de la dépression, ces méthodes nous permettraient de recueillir des données sur la façon dont les facteurs de stress et les transitions vécues par les personnes âgées sont influencées et influencent leur relations avec des personnes importantes dans le réseau, et comment les interactions 
particulières influencent l'humeur, le moral et l'entretien des relations humaines dans le réseau.

Le soutien n'est qu'un des nombreux messages qui peuvent exercer une influence sur le commencement et le déroulement de l'état dépressif. L'évitement, le retrait, la colère, la pitié et le blâme sont d'autres types de messages qui peuvent être communiqués, et ce, malgré les meilleures intentions envers la personne. L'état et le comportement dépressifs peuvent être dérangeants pour les personnes qui veulent aider et sont typiquement difficiles à changer à court terme. C'est pour cette raison qu'il est à la fois décourageant et déprimant de passer beaucoup de temps avec des personnes dépressives. En outre, la présence d'une personne âgée qui est déprimée tend à faire surgir des émotions plus fortes de vulnérabilité et d'impuissance, parce que la majorité des gens associent la vieillesse à la vulnérabilité. Peut-être que l'étude du soutien dans le contexte de la dépression ne doit pas seulement prendre en considération les communications interpersonnelles, mais aussi les notions culturelles reliées à certaines manifestations de communication.

En dernier lieu, nous devrons peut-être nous rappeler que, tout au long de la vie, la dépression passagère est un état transitoire qui peut survenir et qui correspond à un état spécifique dans la gamme des émotions humaines. Il y a des situations où la dépression est l'expression la plus complète et la plus appropriée de nos sentiments, par exemple, lorsqu'elle survient à la suite d'une perte sociale. À une telle occasion, les soins et la compassion sont les meilleures expressions de soutien; il ne s'agit donc pas d'efforts en vue d'atténuer ou d'enrayer la détresse.

\section{Références}

Caplan, G. (1974). Support systems. Dans G. Caplan (Éd.), Support systems and community mental health. New York: Basic Books.

Cassel, J. (1974). Psychological processes and stress. Theoretical formulation. International Journal of Health Services, 4, 471-482.

Cobb, S. (1976). Social support as a moderator of life stress. Psychosomatic Medicine, 38, 300-314.

Gotlib, I.H., \& Hooley, J.M. (1988). Depression and marital distress: Current status and future directions. Dans S. Duck (Éd.), Handbook of personal relationships (pp. 543-570). New York: John Wiley \& Sons.

Rook, K.S. (1984). The negative side of social interaction: Impact on psychological well-being. Journal of Personality and Social Psychology, 46, 1097-1108.

Steinberg, M., \& Gottlieb, B.H. (à venir). Appraisals of spousal support among women facing conflicts between work and family. Dans B. Burleson, T. Albrecht, \& I. Sarason (Éds.), Communicating social support. Newbury Park, CA: Sage Publications. 